\title{
A Depth Measurement System with the Active Vision of the Striped Lighting and Rotating Mirror
}

\author{
Hyongsuk Kim ${ }^{1}$, Chun-Shin Lin $^{2}$, Chang-Bae Yoon ${ }^{1}$, \\ Hye-Jeong Lee ${ }^{1}$, and Hongrak Son ${ }^{1}$ \\ ${ }^{1}$ Division of Electronics and Information Engineering, \\ Chonbuk National Univ., Republic of Korea \\ hskim@moak.chonbuk.ac.kr \\ 2 Department of Electrical and Computer Engineering \\ University of Missouri-Columbia, \\ Columbia, MO 65211 USA \\ Linc@missouri.edu
}

\begin{abstract}
A depth measurement system that consists of a single camera, a laser light source and a rotating mirror is investigated. The camera and the light source are fixed, facing the rotating mirror. The laser light is reflected by the mirror and projected to the scene objects whose locations are to be determined. The camera detects the laser light location on object surfaces through the same mirror. The scan over the area to be measured is done by mirror rotation. Advantages are 1) the image of the light stripe remains sharp while that of the background becomes blurred because of the mirror rotation and 2) the only rotating part of this system is the mirror but the mirror angle is not involved in depth computation. This minimizes the imprecision caused by a possible inaccurate angle measurement. The detail arrangement and experimental results are reported.
\end{abstract}

\section{Introduction}

Active lighting [1-6] techniques have been used extensively in vision applications for depth measurement and 3-D surface reconstruction. Although measuring the distance and orientation of a planar surface using nonstructured lighting [1] is possible, many studies in this area focused on structured lighting. Multiple striped lights [2,3] and rectangular grid of lines [4] are examples of light patterns. The spatial resolution is usually low using multiple or grid of lines. There are also potential ambiguities in matching stripe segments resulting from object surfaces at different depth [7]. An alternative is to use a single light stripe and have it swept over the scene [5][6] by rotating the light projector. One possible drawback of this method is that the image of projected light could be blurred due to its movement. This deteriorates the measurement precision. Another issue arises from the accuracy of the light projection angle. These systems make use of the principle of triangulation to compute depth. For depth much larger than the distance between the camera and the light projector, a small angular error on light projection could cause a significant measurement error. A very precise and reliable measurement of the projection angle can be difficult while the light projector is being rotated. 
In this paper, a setup with the use of a rotational mirror is presented. This system is composed of a single camera, a laser light projector and a rotating mirror. The striped laser light is projected toward the rotational axis of the mirror, and reflected to the surface to be measured. The camera detects the striped light on object surfaces through the same mirror. This arrangement creates several advantages. The first is that potential problems from image blur are eliminated. One special characteristic of this new system is that the light projected to any point (at any direction) at the same horizontal level with the same distance to the mirror axis always forms an image at the same pixel on the camera. Consequently, unless the depth changes over the scanned width, the image of the projected light will not get blurred. This sharp image of the striped light makes necessary image processing easier and measured results more reliable. A blurred image indicates that there is a gradual or abrupt change in depth within the scanned width during one frame of capturing period. The second advantage is that the possible effect from the angular inaccuracy of the light scanning mechanism can be minimized. The only moving part is the mirror. However, the mirror angle is not involved in depth computation. Since the light projector is fixed, only one time of precise calibration for its orientation is required.

\section{The New Depth Measurement System with Striped Lighting}

The new depth measurement system has the single vertical laser light stripe projected to the rotating mirror, and reflected to the scene. The image formed by the same mirror is acquired by the CCD camera. Figure 1 shows the picture of the developed measurement device and the triangulation geometry for the single point projection. Without losing the generality, we focus on the image formation of a single light point. Figure 1(b) shows that the light is reflected by the mirror and projected to an object surface. Note that the mirror can be rotated.

Let the angle between the vertical line and the light source be $\zeta$, and the angle of the mirror from the horizontal axis be $\theta$. Also, let the distance between the camera axis and the rotating axis of the mirror be $\delta_{o}$, the distance between the focal point of the camera and the horizontal axis be $d_{m}$, and the focal distance of the camera be $f$.

The laser light is reflected onto the object at point $\mathrm{T}$ with the mirrored image at $\mathrm{T}$ '. When the mirror angle is $\theta, \angle \mathrm{SOT}$, which is the angle between the projected light and the reflected light, equals $2(\theta-\zeta)$ and the angle $\angle \mathrm{TOM}$ equals $\left(90^{\mathrm{O}}-\theta+\zeta\right)$. Since $\mathrm{T}^{\prime}$ is the mirrored image of $\mathrm{T}$, we have $\angle \mathrm{T}^{\prime} \mathrm{OM}=\angle \mathrm{TOM}=90^{\circ}-\theta+\zeta$.

Consequently, $\angle$ SOT $^{\prime}=2(\theta-\zeta)+\left(90^{\circ}-\theta+\zeta\right)+\left(90^{\circ}-\theta+\zeta\right)=180^{\circ}$. This shows that $\mathrm{T}$ ' will always be on the line along the laser beam, at a distance $R$ from the point $\mathrm{O}$. This characteristic indicates that if the depth of the scanned points during one frame of capturing period is not changed, the image of laser light will remain sharp. Figure 2 illustrates the effect; the projected light point (near the center of the image) is clear while the background gets blurred due to mirror rotation. In image processing, the blurred background actually makes the light point (or stripe) stand out and easy to detect. Note that the part of clear picture at the right is from the scene outside of the mirror. 


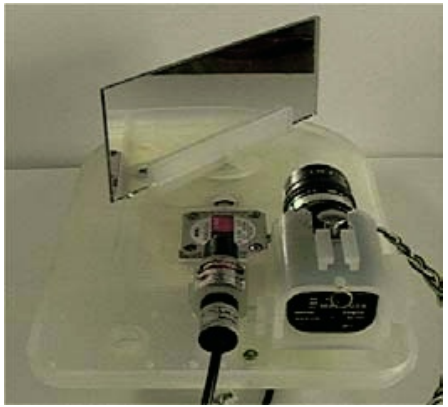

(a) The measurement system

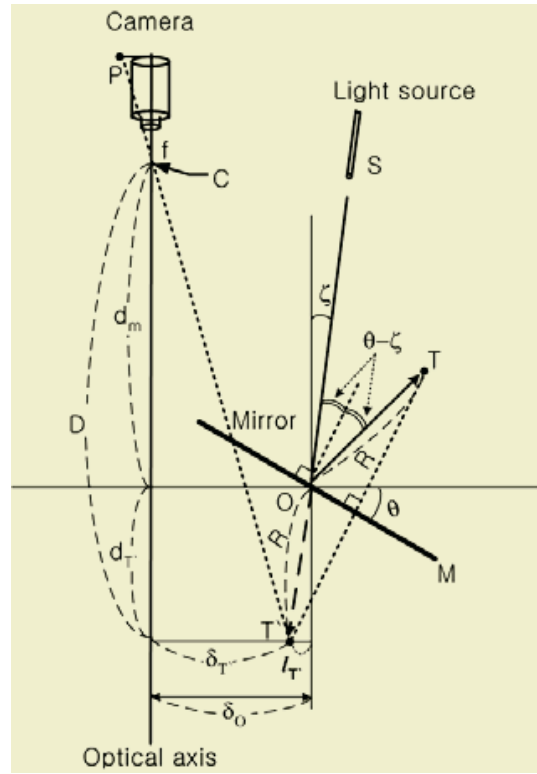

(b) Triangulation geometry for a single point projection

Fig. 1. Depth measurement with light projection and mirror reflection

To derive equations for projection in 3-dimensional space, let's use the cylindrical coordinate system with the mirror axis as the $\mathrm{Z}$-axis. Assume that the light point $\mathrm{T}$ with coordinates $(R, \phi, Z)$ has its image on the CCD sensor at $p=\left(p_{\mathrm{x}}, p_{\mathrm{z}}\right)$ in the coordinates of image plan. Figure 1(b) shows the projection of a point on $x-y$ plane. In this figure, $p_{\mathrm{x}}$ is the distance from $\mathrm{P}$ to the camera optical axis. Using the property of similar triangles, one obtains

$$
p_{x}: f=\delta_{T^{\prime}}: D, \text { where } D=d_{m}+d_{T}
$$

or

$$
p_{x}\left(d_{m}+d_{T^{\prime}}\right)=f \delta_{T^{\prime}} .
$$

Note that $\quad d_{\mathrm{T}^{\prime}}=R \cos \zeta, \delta_{T^{\prime}}=\delta_{O}-l_{T^{\prime}}$ and $l_{T^{\prime}}=R \sin \zeta$. Thus 


$$
p_{x}\left(d_{m}+R \cos \zeta\right)=f\left(\delta_{o}-R \sin \zeta\right)
$$

Solving the above equation for $R$ gives

$$
R=\frac{f \delta_{o}-p_{x} d_{m}}{f \sin \zeta+p_{x} \cos \zeta} .
$$

The angle for the observed point $\mathrm{T}$ is $\phi$, which is defined as the angle measured clockwise from the vertical axis to the line OT. This angle is determined by the laser light direction and the mirror angle as

$$
\phi=2(\theta-\zeta)+\zeta=2 \theta-\zeta .
$$

For the value $Z$, the triangular similarity will give

$$
p_{z}: f=Z: D
$$

or

$$
p_{z}\left(d_{m}+d_{T^{\prime}}\right)=f Z .
$$

Dividing (7) by (2), one obtains

$$
p_{z} / p_{x}=Z / \delta_{T^{\prime}}=Z /\left(\delta_{0}-R \sin \zeta\right) .
$$

Solving the above equation for $Z$ gives

$$
Z=\frac{p_{z}\left(\delta_{0}-R \sin \zeta\right)}{p_{x}}
$$

As a summary, $R, \phi$, and $Z$ can be computed by

$$
\begin{gathered}
R=\frac{f \delta o-p_{x} d_{m}}{f \sin \zeta+p_{x} \cos \zeta} . \\
\phi=2 \theta-\zeta .
\end{gathered}
$$

and

$$
Z=\frac{p_{z}\left(\delta_{0}-R \sin \zeta\right)}{p_{x}} .
$$

Note that the mirror angle is not involved in equation (4) for depth computation. Only the fixed angle $\zeta$ is included and needs to be carefully calibrated. Conceptually, one can consider the 3-D measurement problem as one to determine the position of T', which is the intersection of lines SO and PC (see Figure 1(b)); an error arises when either of those two lines is inaccurately determined. In this setup, the error from inaccurate SO can be minimized by calibrating the angle $\zeta$. Note that for a setup with its laser projector rotated, a measurement error of the projection angle is harder to prevent; so is the depth error. The error from inaccurate PC is caused by inaccurate position of $\mathrm{P}$. Since $\mathrm{P}$ is the pixel position of the light point, a sharper image tends to provide a more precise and reliable result. The characteristic of sharp image illustrated in Figure 2 helps minimize the error from this factor. 


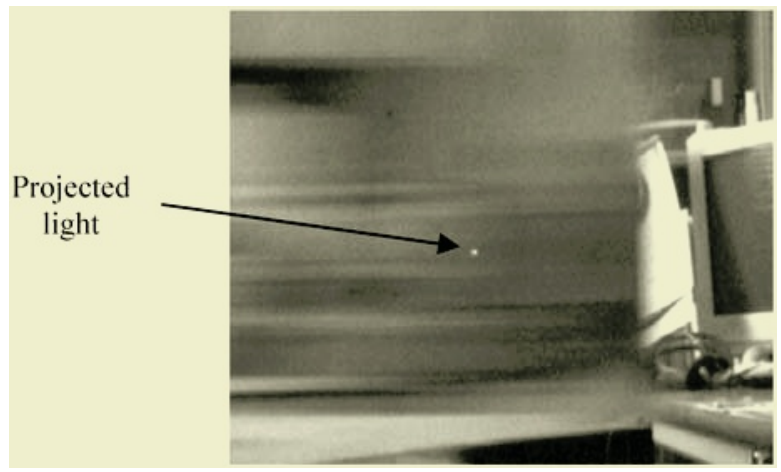

Fig. 2. Laser light point (near the image center) remains sharp while the background is blurred due to mirror rotation

\section{Calibration and Depth Computation}

To use equations (4) to determine the range $R$, system parameters must be either measured or calibrated. In our experiments, $\delta_{0}$ and $d_{\mathrm{m}}$ are measured and known parameters. Other parameters needed include $f$, inter-cell distance $\delta_{\text {cell }}$ on the CCD sensor and the angle $\zeta$. Since the measurement precision is very sensitive to the error of $\zeta$, it is impractical to measure $\zeta$ directly. This parameter must be determined through careful calibration. Precise values of internal parameters of camera such as the inter-cell distance on the CCD plane and the focal length $f$ may not be available and need to be obtained through calibration too.

It is noted that equation (4) can be rewritten such that only the ratio $k=f / \delta_{\text {cell }}$ needs to be calibrated. Let the integer $n_{x}$ be the pixel number corresponding to $p_{x}$, which is the distance from the center of image plane to $\mathrm{P}$. Then $p_{x}$ can be expressed as

$$
p_{x}=\delta_{\text {cell }} n_{x}
$$

Plugging (10) into (4) gives

$$
R=\frac{f \delta_{o}-\delta_{\text {cell }} n_{x} d_{m}}{f \sin \zeta+\delta_{\text {cell }} n_{x} \cos \zeta}=\frac{\frac{f}{\delta_{\text {cell }}} \delta_{o}-n_{x} d_{m}}{\frac{f}{\delta_{\text {cell }}} \sin \zeta+n_{x} \cos \zeta}
$$

Replacing $\frac{f}{\delta_{\text {cell }}}$ by $k$ in (11) results in

$$
R=\frac{k \delta_{o}-n_{x} d_{m}}{k \sin \zeta+n_{x} \cos \zeta}
$$


Depth can be computed using equation (12) after the calibrated values of $k$ and $\zeta$ have been obtained.

\subsection{Calibration of the Internal Parameter $k=\frac{f}{\delta_{\text {cell }}}$}

The camera and the projector can be set up in parallel, i.e., with $\zeta=0$. This is achieved by adjusting the laser light source orientation so that the distance between the laser beam and the camera optical axis at a long distance (e.g., longer than 5 meters) equals $\delta_{0}$. Upon having $\zeta$ set to 0 , experiments can be performed to obtain $n_{x}$ 's for different known ranges of $R$. The collected pairs of $R$ and $n_{x}$ can be plugged into (12) to obtain the estimated values of parameter $k$; the average of these estimated values is used. This parameter needs to be calibrated only once.

\subsection{Calibration of the External Parameter $\zeta$}

For a system with unknown $\zeta$, equation (12) can be used for calibration. One can set up the system to measure a known distance $R$. The value $n_{x}$ can be obtained from image. Values of $\delta_{O}$ and $d_{m}$ are known and $k$ has been calibrated. As a result, the only unknown in (12) is $\zeta$, which can be solved. Since the value of depth is sensitive to the error of angle $\zeta$, recalibration is recommended if the angle is possibly changed.

\section{Experimental Results}

In this section, experimental results are reported. A CCD camera (with 512×480 8-bit pixels) has been used in this study. The distances $d_{m}$ and $\delta_{0}$ were set to $15 \mathrm{~cm}$ and $8 \mathrm{~cm}$, respectively.

\subsection{Calibration of $k$ and $\zeta$}

The method described in Section 3 has been used to determine the constant $k$. The average value of $k$ evaluated at different distances with $\zeta$ set to $0^{\circ}$ is 1377.56 . For depth measurement experiments, $\zeta$ was set approximately to $4^{\circ}$. It was difficult to have $\zeta$ equal exactly to $4^{\circ}$. The precise value had to be obtained from calibration. The system was set up at a distance of $500 \mathrm{~cm}$ from an upright planar surface. The value of $n_{\mathrm{x}}$ for the projected light point was obtained. The actual $\zeta$ was solved from (12) to be $3.869^{\circ}$.

\subsection{Experimental Results on Depth Measurement}

Performance was evaluated for objects at different distances. Figure 3 shows the results for calculations with $\zeta=3.869^{\circ}$ and $\zeta=4^{\circ}$. The result for $\zeta=4^{\circ}$ is provided to show the sensitivity of the precision to $\zeta$ and the importance of good calibration. The 
mirror angle for this experiment was set to $40^{\circ}$. Measurements have also been performed at different directions including $30^{\circ}, 40^{\circ}$ and $50^{\circ}$. Figure 4 shows that the precision is quite consistent for different directions. Note that pixel numbers can only be integer. The quantization seems to be one major factor affecting the precision.

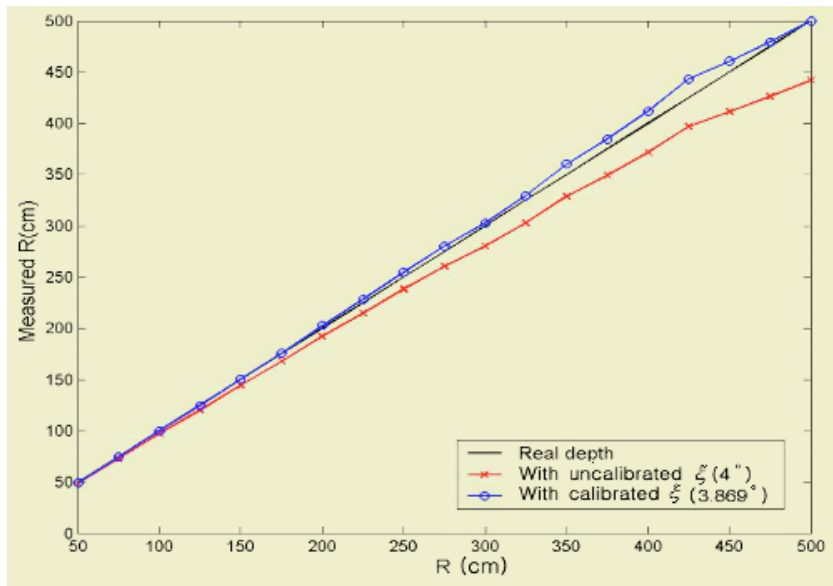

Fig. 3. Results with and without having $\zeta$ calibrated

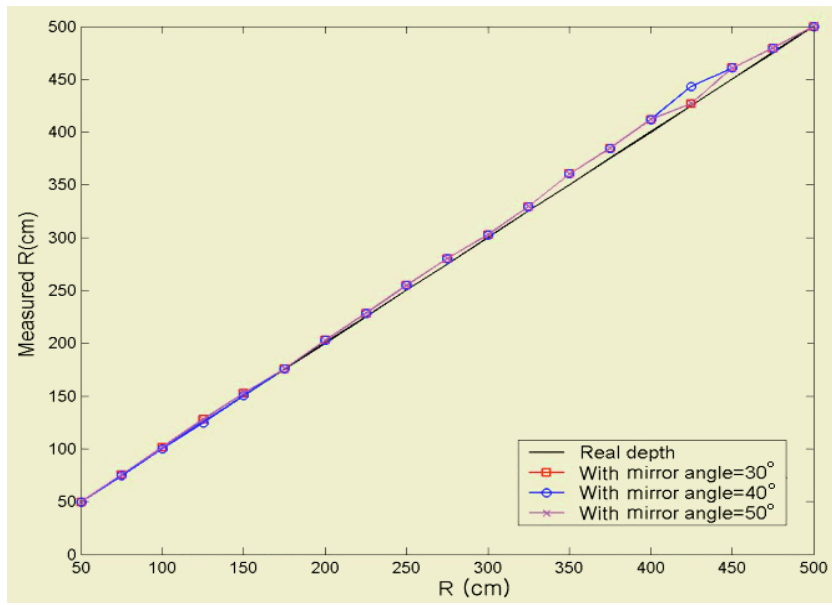

Fig. 4. Plots for measurement at different directions

\section{Conclusions}

A new depth measurement system that consists of a single camera, a laser light stripe projector and a rotating mirror has been investigated. Error analysis provides an idea on the magnitude of expected measurement error. For the distance $400-500 \mathrm{~cm}$, a $20 \mathrm{~cm}$ depth error is expected to be from one-pixel error. Experimental results show a similar magnitude. This arrangement creates several advantages. The first one is that 
the image of the light stripe won't become blurred due to the mirror rotation. The sharpness of the image of light makes necessary image processing easier and measured results more reliable. The second advantage is that the imprecision caused by the inaccurate angle measurement of a laser projector is eliminated with this setup because the light source is not rotated and its angle can be carefully calibrated.

\section{Acknowledgement}

This work has been supported by KESRI(04502), which is funded by MOCIE (Ministry of commerce, industry and energy), and the Korea Institute of Information Technology Assessment.

\section{References}

1. T. Tsukiyama, "Measuring the distance and orientation of a planar surface using nonstructured lighting - 3D measurement system for indoor mobile robots," IEEE Tr. on Instrumentation and Measurement, vol. 45, no. 5, Oct. 1996.

2. V. Srinivasan and R. Lumia, "A pseudo-interferometric laser range finder for robot applications," IEEE Tr. on Robotics and Automation, vol. 5, no. 1, Feb. 1989.

3. M. Baba and T. Konishi, "Range imaging system with multiplexed structured light by direct space encoding," Proceedings of the 16th IEEE Instrumentation and Measurement Technology Conference, vol. 3, pp. 1437-1442, May 24-26, 1999.

4. P. M. Will and K. S. Pennington, "Grid coding: A preprocessing technique for robot and machine vision," Artificial Intelligence, vol. 2, pp. 319-329, 1971.

5. Y. Shirai and M. Suwa, "Recognition of polyhedrons with a rangefinder," in Proc. 2nd Int. Joint Conf. on Artificial Intelligence, pp.80-87, 1971.

6. T. C. Strand, "Optical three-dimensional sensing for machine vision," Optical Engineering, vol. 24, no.1, pp. 33-40, 1985.

7. R. Jain, R. Kasturi and B. G. Schunck, Machine Vision, McGraw-Hill Inc., 1995. 